\title{
Psychological science and analogical reminding in the design of artifacts
}

\author{
THOMAS T. HEWETT \\ Drexel University, Philadelphia, Pennsylvania \\ and \\ BETH ADELSON \\ Rutgers University, Camden, New Jersey
}

\begin{abstract}
In this article, we discuss two approaches to design currently prevalent in the field of humancomputer interaction. Proponents of one approach advocate working from first principles of psychological science; practitioners of the second, engineering, approach find that successful design is often the result of analogical discovery. However, these approaches have not yet been evaluated for the usability of their methods. Can designers work in each of these ways? What are the difficulties? How can they be overcome? In this paper, we take a first step in addressing these questions. We analyze the form in which principle-based design poses its questions, thereby uncovering some of the difficulty that is encountered in this way of working. Furthermore, we analyze the form in which engineering problems are expressed and identify an advantage of this typically analogical way of working. We then suggest a way in which analogy-based design can supplement both principle-based and empirically driven design.
\end{abstract}

In this article, we discuss two approaches to design currently prevalent in the field of human-computer interaction (HCI): derivations from first principles and analogical retrieval. Proponents of one approach advocate working from first principles of psychological science; practitioners of the second, engineering, approach find that successful design is often the result of analogical discovery. In the approach motivated by science-based design, first principles are used to guide artifact development (Carroll, 1991; Carroll, Kellogg, \& Rosson 1991; Carroll \& Rosson, 1995). This approach is practiced within the context of the fundamental question of science: What is the generalized abstraction describing the mechanism underlying phenomenon $X$ ? In physics, the science question provides an answer of the form: Force $=$ Mass $X$ Acceleration. Work $=$ Mass $\times$ Distance. These statements are extremely powerful in that they provide broadly applicable solutions to large classes of problems.

In the second approach to design considered here, past problem solutions are retrieved from memory and are used

\footnotetext{
Work on this paper was supported in part by NSF CISE Grant CCR9527130 to T.T.H. The authors thank Marilyn Mantei and the anonymous reviewers for comments on an earlier draft of this paper. The authors also thank Jack Carroll, Mary Beth Rosson, Tom Landauer, Dedre Gentner, Paul Thagard, and Keith Holyoak for stimulating discussions about the issues in this paper. Correspondence should be addressed to T. T. Hewett, Department of Psychology/Sociology/ Anthropology, Drexel University, Philadelphia, PA 19104 (e-mail: hewett@drexel.edu).
}

as analogies to solve current problems. The second approach is practiced within the context of the fundamental question of engineering: How can I build a mechanism that exhibits behaviors $X$ through $Z$ ? The form of each question facilitates a particular method of problem-solving. The form of the engineering question, How can I build a mechanism that exhibits behaviors $X$ through $Z$ ? provides specifications of the behaviors that the to-bedesigned artifact must exhibit. These specifications can be used as retrieval cues for mechanisms already known to the designer, since past design solutions are often encoded with respect to the behaviors they exhibit. The posing of the "How can I build. .." question therefore tends to lead to design by analogy. Evidence for the importance of analogical transfer in design thinking can be found in a variety of sources (e.g., Candy \& Edmonds, 1994), and our review of the literature finds evidence of working by analogy across a wide range of areas of human endeavor that can be seen as related to or involving design in some way (e.g., Burstein, 1983, 1986; Carbonell, 1983, 1986; Clement, 1988; Dunbar, 1995; Hewett \& DePaul, in press; Holyoak \& Thagard, 1995; Kedar-Cabelli, 1984).

\section{EXAMPLES OF TWO DESIGN APPROACHES}

In this section of the paper, we discuss an example of working by analogy and an example of how analogical reminding influences design. As a result of the frequent occurrence of analogical reasoning, we focus on the strength of this method. Next, we provide an example of 


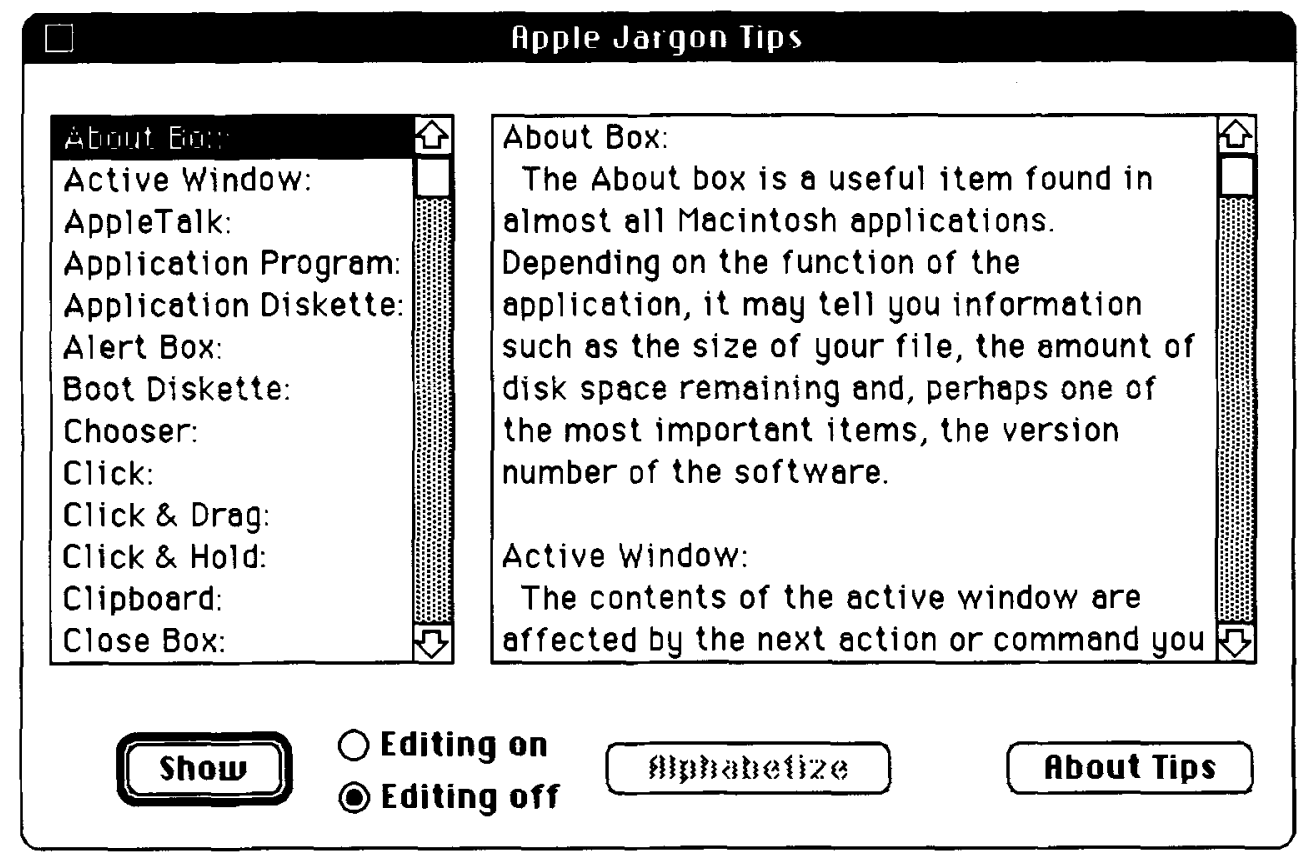

Figure 1. Drexel Disk tips window.

the potential weakness in working from first principles. In the final section of the paper, we discuss the need for a balanced evaluation of each of the approaches presented.

\section{Designing From Analogy: Getting to First Principles via Previous Cases}

To illustrate a design that was generated by analogy and that worked well because of its concordance with underlying psychological first principles, consider a software package whose success can, in part, be explained by the encoding specificity principle. The screen display in Figure 1 is from the Drexel Disk (Hewett, Perkey, \& Boardway, 1988). The Drexel Disk was first developed in 1984 as an information resource for students at Drexel University, where all students are required to have access to a personal computer. A more complete description of the Drexel Disk and the design guidelines used in its development can be found in Hewett (1989).

The screen in Figure 1 (Apple Jargon Tips) shows a glossary of Macintosh-related terms. Also included on the disk were similar files that provided information on spreadsheet use, word processing, printing, and a variety of other useful things that could be done with the Macintosh and its software. The use of each "tip" file is the same. On the left side of the screen appears a box containing a list of key terms; on the right are the same terms and their associated explanations. The user is able to browse through either the term list or the descriptions themselves. While browsing through the term list on the left, if one selects a term and clicks on the Show button, that action activates a search in which the term is used by the system as a retrieval cue, and, as in the screen in Figure 2, the appropriate description is brought into view at the top of the box on the right. Thus, when searching for particular information, one can scroll through the list of key terms until the desired one has been found and then have the computer retrieve the full description.

The design of the system is such that the user has a choice, however. If the key term itself is a sufficient cue to allow the user to recall the associated description, the interaction can end there; if the term is not a sufficient cue, the system, at the user's request, performs the task for the user. The point here is that the user is not forced into an unnecessarily extended dialogue and then asked to scroll through and read a large amount of already understood text. That this is a pleasing design choice becomes clear when it is contrasted with the familiar case in which the user is told that a system error has occurred and the machine is inexorably going to crash but the user is nevertheless forced to consent to the crash through a mouse click on an OK button.

Two factors may contribute to the user's ability to recall a term's description. The first has to do with the semantic match between the term and the description; the second has to do with the episodic match. The semantic features of a term may be sufficient to allow the term's recall. For example, looking at the second term in the term list in Figure 2, "Quickeys" may remind the user that certain keys allow the user to quickly specify a command from the keyboard, obviating a (perhaps nested) menu 


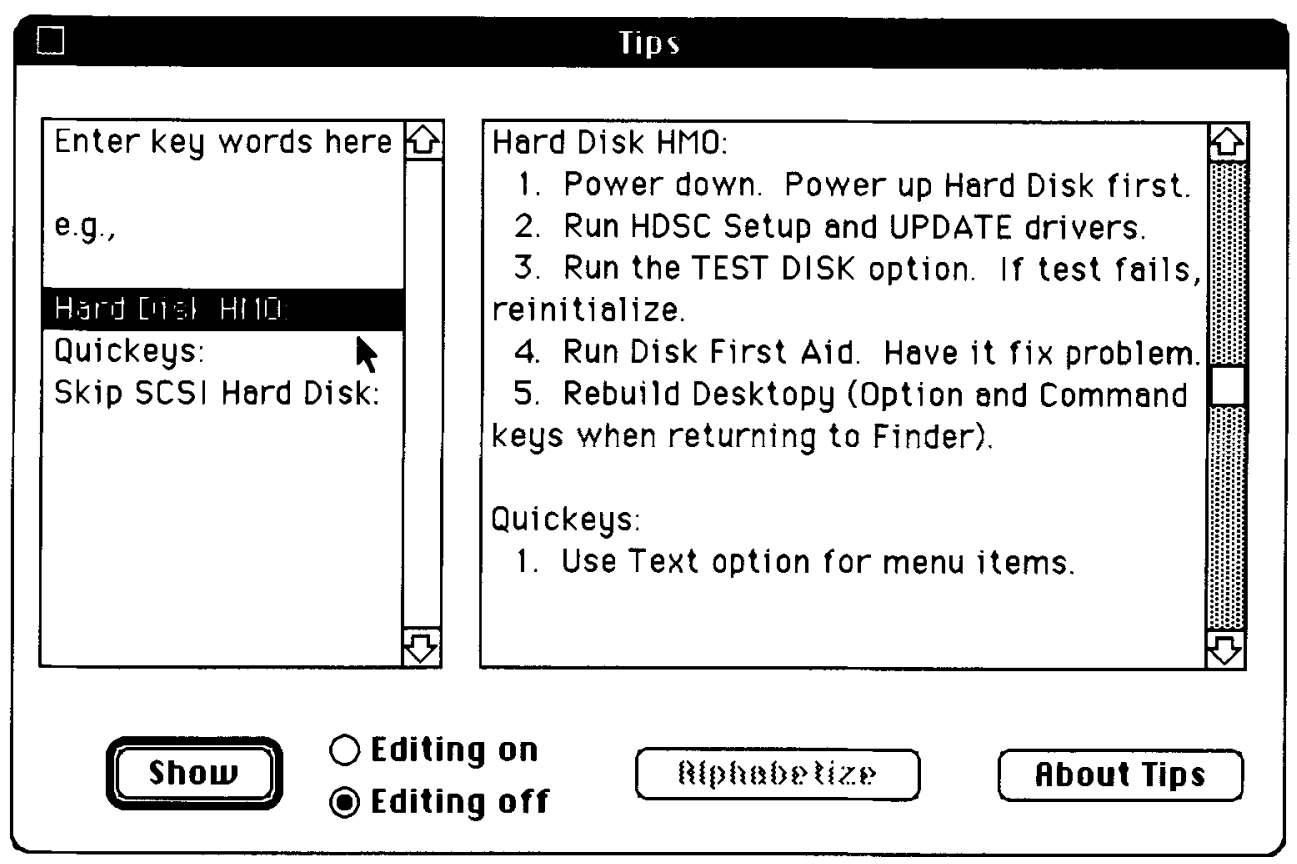

Figure 2. Activating a key search.

search and selection. However, even when retrieval is being aided by semantic specificity, episodic elements can enter into the process (Roediger \& Adelson, 1980).

In their work on encoding specificity, Tulving, Thomson, and colleagues (e.g., Thomson, 1972; Thomson \& Tulving, 1970; Tulving, 1974; Tulving \& Osler, 1968; Tulving \& Thomson, 1973; Tulving \& M. J. Watkins, 1975; Tulving \& O. C. Watkins, 1977) have shown that the cues present and the organization imposed upon material at the time of learning, influence its later accessibility. If the context of learning can be reinstated, retrieval is improved. In the current context, the encoding specificity principle tells us that a term evocative of the learning context can lead a user to recall the term's associated description. In the case of the Drexel Disk, the terms on the left are intended to function both as keys for the computer's search and as reinstating cues for the user. That is, the designers of Drexel Disk allowed for the possibility that the episodic properties of the terms might allow users to recall the associated information on their own. At this point, we want to stress that, although the designers of Drexel Disk wanted users to benefit from encoding specificity effects, they came to this position through an analogical insight, rather than through a direct intention.

Not long before beginning the original design of the Drexel Disk, one of the designers found himself searching for instructions on how to rebuild the desktop of his Macintosh. He knew he had previously seen the instructions in a back issue of a particular publication, and he remembered which publication, but he had forgotten the issue. He went to his bookshelf with the intention of performing a serial exhaustive search, starting at the first issue for the current year. However, he found that the act of searching reinstantiated the situation in which he first encountered the solution to his problem. The reinstantiation included the time of year, allowing him to go directly to the correct issue.

In thinking about the design problem of providing users quick, simple access to Macintosh tips to be included in the information structure of the Drexel Disk, the designer had a minor "ah ha" experience. He realized that users of Drexel Disk might have the same episodically based reminding that he had experienced in searching for the desktop rebuilding tip, and he decided to allow for this in his design. For example, simply finding the reminder to "Buy flowers" in one's to-do file (see Figure 3) might well lead the user to recall that roses are the flowers of the recipient's choice.

We want to stress that although the information retrieval analogy allowed Hewett et al. (1988) to capitalize on the efficiency of the encoding specificity effect, the solution was arrived at via analogy rather than derivation. The designers of the Drexel Disk were faced with the engineering question, How can I build a mechanism that has functionality $\mathrm{X}$ through $\mathrm{Z}$ ? The explicit stating of the desired functionalities made it possible to recognize an analogous problem with a desirable solution. That is, past designs are often encoded with respect to their functionality, and so the desired functionality served as an appropriate "memory index" allowing the designers to recognize that the journal search problem held a key toward the design of the Drexel Disk (Adelson, 1990). As described earlier, engineers often work from analogy rather than from first principles. A cantilevered beam 


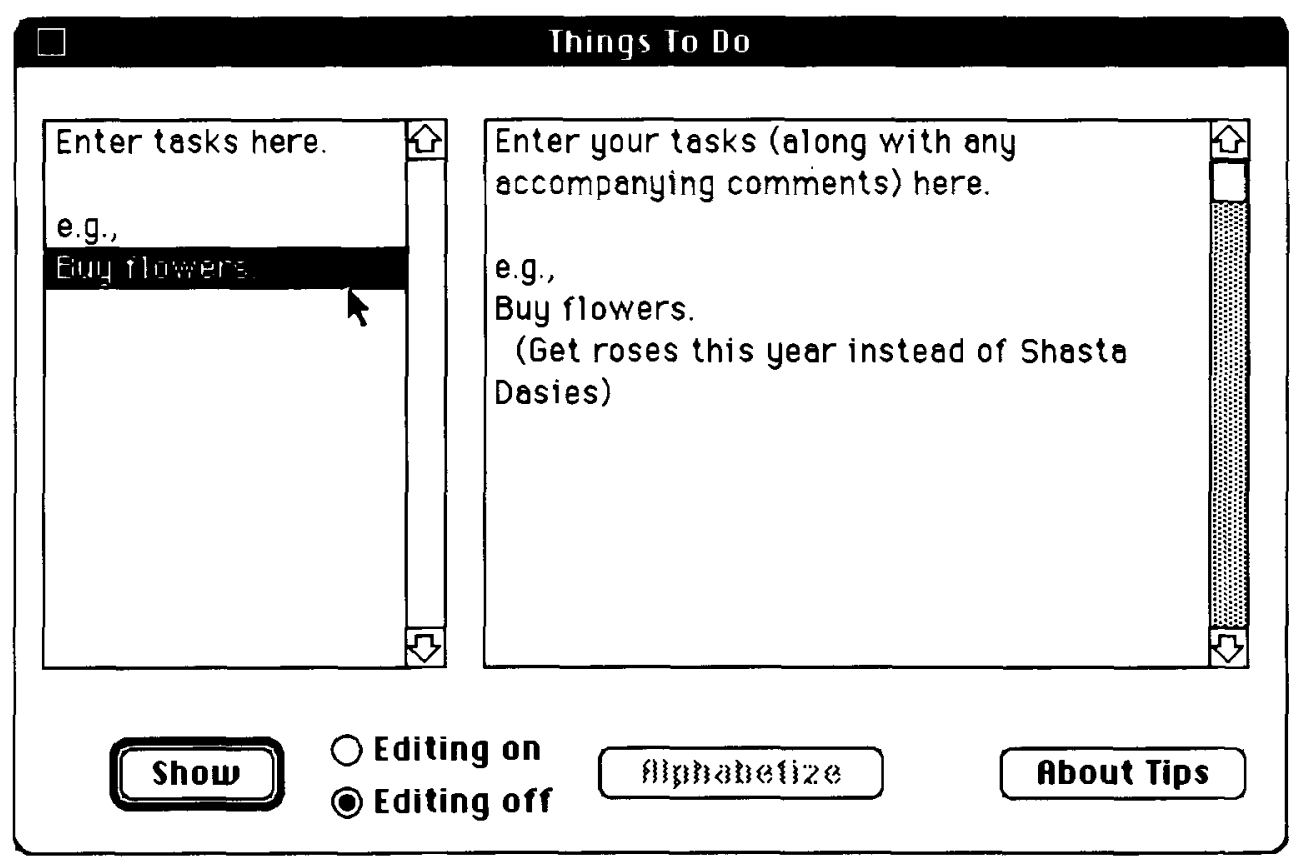

Figure 3. A to-do list reminder.

provides an analogy for a cantilevered bridge, as does a suspension bridge for a suspension building. An inclined plane provides an analogy for a wheel.

Consider the following examples, which, although retrospective, are nevertheless illustrative of the phenomenology of analogical design. According to Samuel Morse's diaries (Okagaki \& Koslowski, 1983), initially, in trying to transmit telegraphic signals across significant distances, Morse tried the strategy of building successively stronger generators. He found, however, that the signals still degraded with distance. Supposedly, the solution to the problem came to him in the following way. While riding on a train, he happened to look out of the window and notice a Pony Express depot, at which horses were being fed and watered. Morse realized that the relay station strategy constituted an analogical solution to the telegraph problem as well. In a similar vein, Edison's diaries recount that he invented the kinetiscope by setting out to do for the eye what he had done for the ear with the phonograph (Brian, 1926).

Working from a problem statement that specifies the behavioral requirements of the solution allows the designer to find analogous mechanisms that can be appropriately applied or adapted. By contrast, working from principles that do not contain statements about contexts of application can be an error-prone process, as we see in the short-term memory (STM) example described below. The reason that designing from first principles is sometimes difficult stems from the nature of first principles and the resulting way in which they are stated. As stated earlier, a first principle is a generalized abstraction describing a given phenomenon: Force $=$ Mass $\times$ Acceleration . Work $=$ Mass $\times$ Distance. In psychology, we have a for- malization that describes a characteristic of STM: STM capacity $=7 \pm 2$ chunks.

Although $F=$ MA provides a definition of force, and STM capacity tells us only one of the features of the store and is not a full definition, the important commonality is that both statements are decontextualized. These decontextualized general statements assume but do not make explicit the range of cases to which they apply. In the formulation of Work, a frictionless state is assumed. In the formulation of STM capacity, it is assumed that items can be either atoms or compound chunks and that no external memory aids are present. The contextualizing information that tells the designer when the use of STM capacity limitations is an appropriate proviso is absent, and so it is difficult to use the abstract statement to either guide or constrain prescriptions for action.

Furthermore, although problem specifications that are stated in terms of sought behaviors do facilitate the retrieval of analogous cases, they do not provide cues leading directly to the retrieval of relevant first principles. This suggests a reason why the engineering question does not lead to design from first principles. The first principles are generalized, and therefore decontextualized, statements, and so there is a mismatch between the formulation of the cue and the target.

\section{Designing From First Principles: \\ Figuring Out What Principle to Apply When}

At a university that will remain nameless, there are classes in which students are taught about HCI. In some classes, students are given the following design guideline: "The Macintosh interface is a bad interface because there are menus with more than seven items," the implication 
being, "Do not design interfaces like that of the Macintosh." The logic of the argument, both explicit and implicit, runs roughly as follows. Miller (1956) showed that the capacity of STM is $7 \pm 2$. Exceeding the capacity of STM degrades recall performance. As noted, the Macintosh has menus with more than 7 items. Therefore, an interface such as the one found on the Macintosh will be a bad interface because the menus exceed the capacity of STM and will lead to degraded performance.

There are, however, three problems that arise from applying this guideline to this design choice. First, menu items are generally related (e.g., File, Edit, Format, or View). That is, the contents of a menu tend to form a single chunk rather than a set of greater than 7 unrelated items. Second, menu titles are generally mnemonic and so provide effective recall cues for the items within. For both these reasons, menu titles aid rather than stress the user. Additionally, once the menu has been pulled down, the contained elements are now "knowledge in the world" (Norman, 1988) rather than in the head. None of the items must be stored in working memory. Using Miller's work to proscribe the design of graphical user interfaces with more than 7 items per menu is misleading at best and is analogous to claiming that a penny has a bad interface because people have trouble recalling all of the information on its face (Nickerson \& Adams, 1979).

While presented in the particular, this example is not an isolated case. Several people have made this or similar claims about the implications of Miller's (1956) work for $\mathrm{HCI}$. But what is important about this case is that it represents an attempt to get students to design from first principles. As stated above, the reason that this is difficult stems from the nature of first principles and the resulting way in which they are stated. The statement "STM capacity $=7 \pm 2$ " assumes but does not state the range of cases to which it applies. Nowhere is it made explicit that items can be either atoms or chunks and that capacity is an issue only when external memory aids are not present. The contextualizing information that tells the designer when Miller's work is appropriate is absent, and so it is hard to use the abstract principle to either guide or constrain prescriptions for action.

\section{CONCLUDING REMARKS}

Reflection on the two examples provided above suggests a fundamental difference in orientation and approach. In the first case, the designer focused on a particular design problem and brought an analogous case to bear within the conditions created by the problem context, thereby capitalizing on the psychological principle contained in the analog. In the second case, the instructor focused on a generally applicable, decontextualized rule and sought to use it to guide design.

The two examples discussed here have been used to illustrate one of the benefits of (and perhaps underlying reasons for) designing by analogy as well as one of the difficulties in designing from first principles. What we need now is a balanced account. Analogy has its benefits but also its drawbacks. As discussed elsewhere, analogies are, definitionally, imperfect problem solutions that need debugging, pruning, and/or extension (Adelson, 1989, 1996; Adelson et al., 1988; Burstein \& Adelson, 1987, 1992). On the other hand, the work of Carroll, Rosson, and colleagues (Carroll, 1991; Carroll et al., 1991; Carroll \& Rosson, 1995) working from first principles has led to a number of notable "HCI success stories."

In our current work, we are beginning to unpack the range of benefits and difficulties associated with both ways of working. At the current time, what we can begin to do is place the examples presented here in the context of other recent work by Carroll and his colleagues and by Landauer and his colleagues (Landauer, 1991, 1995).

In his work on the requirements for an "active" or applied science of design, Carroll (1991) stresses that such a science must be both specific and applicable. That is, the science must relate to both the content and the practice of design. He goes on to suggest that a special level of description and/or special vocabulary of description may be required. In addition, he notes that this level or vocabulary of description may be couched in a form that is not reducible to the starting science.

We suggest that if this metalevel is to express itself in a language compatible with practice, it may take the form of analogs. In fact, analogs may work very well. Carroll suggests that designers should work from systematically enumerated sets of scenarios of use, to causal explanations of the results of the scenarios ("claims"), to principled redesign (Carroll et al., 1991; Carroll \& Rosson, 1995). Carroll points out that going from claims to redesign is difficult. However, claims generate explanations that tell us what behaviors were desired but not achieved.

As stated above, once we have a description of a desired behavior, we have a retrieval cue for an analogous solution. Analogies then are expressed in a language compatible with observed practice and can form the bridge between claims and redesign. Additionally, meeting another criterion for good applied science, analogies allow reuse.

Landauer (1991) takes a position that prescribes an approach to $\mathrm{HCI}$ design different from that proposed by Carroll-an approach in which design is guided not by science but by experimentation. Landauer too is able to report "HCI success cases" (see Landauer, 1995). The wide divergence of opinion between the bodies of work of Carroll and Landauer suggests that a rather fundamental issue has not yet been surfaced and clarified. Interestingly, Landauer's $(1991,1995)$ work also reveals a role for the analogical problem solving we have seen and described here. For example, Landauer (1987) argues that a careful, disciplined empirical investigation can easily reveal what people cannot do well and suggest ways a computer might help. He further notes that he believes 
that the step between an understanding provided by empirical studies and the creation of new cognitive tools can sometimes be short.

We suggest that empirical studies also tell designers what desired behaviors were not achieved. Again, if the results of empirical studies are formulated in these terms, analogies can be used to suggest useful and usable tools. In closing, we expect that the further explication of the role of analogical reminding in design will contribute to an enhanced understanding both of HCI design and of design in general.

\section{REFERENCES}

ADELSON, B. (1989). Uncovering how designers design. Research in Engineering Design, 1, 35-42.

Adelson, B. (1990). Recognizing novel uses for familiar plans. In Proceedings of the Twelfth Annual Meeting of the Cognitive Science Society (pp. 253-260). Hillsdale, NJ: Erlbaum.

ADELSON, B. (1996). Characterizing analogical reasoning. In M. Waldron \& K. Waldron (Eds.), Mechanical design: Theory and methodology (pp. 240-263). New York: Springer-Verlag.

adelson, B., Gentner, D., Thagard, P., Holyoak, K., Burstein, M., \& HAMMOND, K. (1988). The role of analogy in a theory of problemsolving. In Proceedings of the Tenth Annual Meeting of the Cognitive Science Society (pp. 298-304). Hillsdale, NJ: Erlbaum.

BuAN, G. (1926). Edison: The man and his work. Garden City, NY: Knopf.

BuRStEIN, M. H. (1983). Causal analogical reasoning. In R. S. Michalski, J. G. Carbonell, \& T. M. Mitchell (Eds.), Machine learning: An artificial intelligence approach (Vol. 1, pp. 162-182). Los Altos, CA: Morgan Kaufmann.

BURSTEIN, M. H. (1986). Concept formation by incremental analogical reasoning and debugging. In R. S. Michalski, J. G. Carbonell, \& T. M. Mitchell (Eds.), Machine learning: An artificial intelligence approach (Vol. 2, pp. 351-369). Los Altos, CA: Morgan Kaufmann.

BuRSTEIN, M. [H.], \& ADELSON, B. (1987). Mapping and integrating partial mental models. In Proceedings of the Ninth Annual Meeting of the Cognitive Science Society (pp. 11-22). Hillsdale, NJ: Erlbaum.

Burstein, M. [H.]. \& Adelson, B. (1992). Analogical reasoning for learning. In R. Freedle (Ed.), Applications of artificial intelligence to educational testing (pp. 137-172). Hillsdale, NJ: Erlbaum.

CANDY, L., \& EDMonds, E. A. (1994). Artifacts and the designer's process: Implications for computer support to design. Journal of Design Sciences \& Technology, 3, 11-31.

Carbonelt, J. G. (1983). Transformational analogy, problem solving and expertise acquisition. In R. S. Michalski, J. G. Carbonell, \& T. M. Mitchell (Eds.), Machine learning: An artificial intelligence approach (Vol. 1, pp. 137-161). Los Altos, CA: Morgan Kaufman.

CarbonelL, J. G. (1986). Derivational analogy: A theory of reconstructive problem solving and expertise acquisition. In R. S. Michalski, J. G. Carbonell, \& T. M. Mitchell (Eds.), Machine learning: An artificial intelligence approach (Vol. 2, pp. 372-392). Los Altos, CA: Morgan Kaufmann.

Carroll, J. M. (1991). Introduction: The Kittle House manifesto. In J. M. Carroll (Ed.), Designing interaction (pp. 1-16), Cambridge: Cambridge University Press.

Carroll, J. M., KellogG, W. A., \& Rosson, M. B. (1991). The taskartifact cycle. In J. M. Carroll (Ed.), Designing interaction (pp. 74102). Cambridge: Cambridge University Press.

Carroll, J. M., \& Rosson, M. B. (1995). Getting around the task- artifact cycle: How to make claims and design by scenario. In M. Rudisill, C. Lewis, P. B. Polson, \& T. D. McKay (Eds.), Human-computer interface design (pp. 229-268). San Francisco: Morgan Kaufmann. Clement, J. (1988). Observed methods for generating analogies in scientific problem-solving. Cognitive Science, 12, 563-586.

DUNBAR, K. (1995). How scientists really reason: Scientific reasoning in real-world laboratories. In R. J. Sternberg \& J. Davidson (Eds.), The nature of insight (pp. 365-395). Cambridge, MA: MIT Press.

HEWETT, T. T. (1989). The Drexel Disk: Hypertext-based instructional software as a tool for the exploration of a constrained knowledge space. Behavior Research Methods, Instruments, \& Computers, 21 , 316-325.

HewetT, T. T., \& DePaul, J. L. (in press). Toward a human centered scientific problem solving environment. In E. Houstis, R. Bramley, \& S. Gallipolous (Eds.), Scientific problem solving environments. Los Alamitos, CA: IEEE Press.

Hewett, T. T., Perkey, M. N., \& Boardway, L. (1988). The Drexel Disk 4.0 [Computer Software]. Philadelphia, PA: Drexel University.

Holyoak, K., \& ThaGaRD, P. (1995). Mental leaps. Cambridge, MA: MIT Press.

KedAR-CaBeLLI, S. (1984), Analogy with purpose in legal reasoning from precedents (Tech. Rep. No. 17). New Brunswick, NJ: Rutgers University, Laboratory for Computer Science.

LANDAuER, T. (1987). Psychology as a mother of invention. In S. Pemberton (Ed.), Human factors in computing systems (CHI ' 97 Conference Proceedings, pp. 333-335). New York: Association for Computing Machinery.

LANDAUER, T. K. (1991). Let's get real: A position paper on the role of cognitive psychology in the design of humanly useful and usable systems. In J. M. Carroll (Ed.), Designing interaction (pp. 60-73). Cambridge: Cambridge University Press.

LANDAUER, T. K. (1995). The trouble with computers. Cambridge, MA: MIT Press.

MILLER, G. A. (1956). The magical number seven, plus or minus two: Some limits on our capacity for processing information. Psychological Review, 63, 81-97.

NiCKerSON, R. S., \& ADAMS, M. J. (1979). Long-term memory for a common object. Cognitive Psychology, 11, 287-307.

Norman, D. A. (1988). The psychology of everyday things. New York: Basic Books.

OKAGAKI, L., \& KosLowsKI, B. (1983). Another look at analogies and problem-solving. Journal of Creative Behavior, 21, 11-23.

Roediger, H. L., III, \& Adelson, B. (1980). Semantic specificity in cued recall. Memory \& Cognition, 8, 65-74.

Thomson, D. M. (1972). Context effects in recognition memory. Journal of Verbal Learning \& Verbal Behavior, 11, 497-511.

Thomson, D. M., \& Tulving, E. (1970). Associative encoding and retrieval: Weak and strong cues. Journal of Experimental Psychology, 86, 255-262.

TuLVING, E. (1974). Recall and recognition of semantically encoded words. Journal of Experimental Psychology, 102, 778-787.

TULVING, E., \& OSLER, S. (1968). Effectiveness of retrieval cues in memory for words. Journal of Experimental Psychology, 77, 593-601.

Tulving, E., \& Thomson, D. M. (1973). Encoding specificity and retrieval processes in episodic memory. Psychological Review, 80, 352-373.

Tulving, E. \& Watkins, M. J. (1975). Structure of memory traces. Psychological Review, 82, 261-275.

TUlVING, E., \& WaTkINS, O. C. (1977). Recognition failure of words with a single meaning. Memory \& Cognition, 5, 513-522.

(Manuscript received October 21, 1997; revision accepted for publication February 9, 1998.) 